\title{
Neutrophil gelatinase-associated lipocalin protein as a biomarker in the diagnosis of breast cancer: A meta-analysis
}

\author{
$\mathrm{YU}_{\mathrm{WANG}}{ }^{1}$ and TINGTING ZENG ${ }^{2}$ \\ ${ }^{1}$ Laboratory of Molecular Diagnosis of Cancer, State Key Laboratory of Biotherapy and Cancer Center, \\ West China Hospital, Sichuan University, Chengdu, Sichuan 610041; ${ }^{2}$ Adverse Drug Reaction Monitoring Center of Chengdu, \\ High-Tech District, Chengdu, Sichuan 610042, P.R. China
}

Received January 5, 2013; Accepted March 12, 2013

DOI: $10.3892 /$ br.2013.89

\begin{abstract}
Neutrophil gelatinase-associated lipocalin (NGAL) has been thought to play an important role in breast cancer tumorigenesis and progression. Various studies have focused on the association between NGAL and breast cancer. The aim of this meta-analysis was to establish the overall accuracy of the NGAL test in the diagnosis of breast cancer. A comprehensive search of the literature was conducted using PubMed, OVID, ScienceDirect and the China National Knowledge Infrastructure (CNKI) databases, and our screening covered all published papers until November 2012. The relevant papers were selected according to stringent inclusion criteria. Essential data were abstracted from the included papers and further analysed by a systematic meta-analysis. The present meta-analysis included four study papers. The summary estimate was 64\% (95\% CI, 0.59-0.69) for sensitivity and $87 \%$ (95\% CI, 0.81-0.92) for specificity. The positive likelihood (PLR), negative likelihood (NLR) and diagnostic odds (DOR) ratios were 5.63 (95\% CI, 3.63-8.74), 0.32 (95\% CI, 0.14-0.71) and 18.02 (95\% CI, 9.84-32.98), respectively. The area under the summary ROC curve (AUC) for the diagnosis of breast cancer was 0.9008 . Thus, NGAL is a potential biomarker for the diagnosis of breast cancer.
\end{abstract}

\section{Introduction}

Breast cancer is a malignancy that affects women worldwide. Despite developments in surgery and chemotherapeutics, early diagnosis and prognosis of breast cancer remain poor. Previous studies have investigated Muc-1 gene-related protein biomarkers CA15.3 and CA27.29 (1,2), Her-2/neu (3) and CEA (4), however, each of these potential biomarkers has

Correspondence to: Dr Tingting Zeng, Adverse Drug Reaction Monitoring Center of Chengdu, High-Tech District, 366 Jincheng Avenue, Chengdu, Sichuan 610042, P.R. China

E-mail: bettyzeng@foxmail.com

Key words: neutrophil gelatinase-associated lipocalin, lipocalin-2, breast cancer, diagnosis, meta-analysis limitations with regard to sensitivity, specificity or the scale of applicability. At present, no single protein serves as a biomarker for the screening of breast cancer. The combined application of biomarkers in biochemical detection, such as CEA-TPA-CA15.3 (5), ameliorates the weakness of each biomarker. However, the cost and complicated evaluation process involved in the detection of this type of cancer suggests a combined application is not feasible. Therefore, it is crucial to identify biomarkers with high diagnostic value.

Neutrophil gelatinase-associated lipocalin (NGAL) also known as lipocalin 2 is a $25-\mathrm{kDa}$ glycoprotein, which was originally identified as a covalent complex with matrix metalloproteinase-9 (MMP-9) in human neutrophil $(6,7)$. NGAL has recently been investigated in a variety of physiological and pathological conditions. As a biomarker, the diagnostic value of NGAL has been identified in acute kidney injury (8). Additionally, NGAL is involved in various types of human cancer. NGAL expression is upregulated in the majority of human cancers, including colorectal neoplasia (9-11), gastric cancer (12), oesophageal squamous cell carcinoma (13), lung adenocarcinoma (14), primary liver carcinoma (15) and thyroid neoplasia (16). However, this expression is downregulated in cancers such as pancreatic (17-19) and prostate (20), as well as chronic myeloid leukemia $(21,22)$.

The NGAL gene in human is highly expressed in luminal epithelial cells compared to myoepithelial cells (23). Subsequently, the majority of breast carcinomas were thought to develop from luminal epithelial cells. Thus, NGAL may actively participate in breast cancer progression (24). NGAL levels were also strongly correlated with poor histological grading, lymph node metastasis, high carcinoma proliferation ability and weak prognosis of breast cancer patients (25).

However, no specific study has sufficiently evaluated the correlation between NGAL expression and the risk of breast cancer. Therefore, in the present study, we aimed to estimate the possibility of NGAL as a biomarker in the early diagnosis of breast cancer via a meta-analysis of published literatures.

\section{Materials and methods}

Data sources and search strategy. The meta-analysis of Observational Studies in Epidemiology (MOOSE) guidelines for the conduct of meta-analyses of observational 
cohort studies were followed. Two invesgators (Y.W. and T.T.Z.) conducted a literature search using PubMed, OVID, ScienceDirect and the China National Knowledge Infrastructure (CNKI) databases, including all published papers until November 2012 using a combination of the following terms: neutrophil gelatinase-associated lipocalin, NGAL, Lipocalin 2 and breast cancer. There were no language restrictions.

Publication selection. The investigators Y.W. and T.T.Z. independently reviewed potentially associated publications by checking their titles and abstracts and then procured the most relevant papers for further examination. Moreover, the reference lists of the selected studies were also screened for any potential information. The criteria used to select studies for the meta-analysis were: i) studies focusing on the association of NGAL with breast cancer; ii) observational studies; iii) studies that reported breast cancer pathological diagnoses and sources of cases and controls; iv) test methods [immunohistochemistry (IHC) or ELISA] and v) completeness of data, or availability of information that proved useful in deriving results. Exclusion criteria included: i) different design and definition of experiments; ii) source of cases and controls and other important information could not be obtained; iii) animals or in vitro experiments; iv) reviews and repeated literature.

Data extraction and study quality assessment. Data including author, publication year, region, study population and the measurement method of NGAL were extracted by two independent reviewers (Y.W. and T.T.Z.) and entered into a database. For conflicting evaluations, agreement was achieved following a discussion. The quality of each included study was assessed using the diagnostic accuracy tool, quality assessment for studies of diagnostic accuracy (QUADAS; maximum score, 14).

Statistical analysis. The studies were analysed using a Chi-square-based Q statistic test to assess heterogeneity and $\mathrm{I}^{2}$ to estimate the degree of heterogeneity. Statistically significant heterogeneity was considered when $\mathrm{P}<0.05$ and the $\mathrm{I}^{2}$ value was $>50 \%$. If there was significant heterogeneity, the random-effect model (DerSimonian and Laird) was used. Otherwise, the fixed-effect model (Mantel-Haenszel) was employed.

The bivariate model was applied for the diagnostic meta-analysis in order to perform the pooled sensitivity, specificity, as well as the positive likelihood (PLR), negative likelihood (NLR) and diagnostic odds (DOR) ratios. Pooled estimates with the corresponding 95\% CI were initially calculated using the appropriate statistical analysis model. In addition, summary receiver operator characteristic (sROC) curves were constructed. The area under the curve (AUC) value with the Q-value was also calculated to present an overall summary of test performance in order to differentiate between a diseased and non-diseased participant. Spearman's correlation coefficient of sensitivity and 1-specificity was calculated to estimate the threshold effect. The publication bias of included studies was assessed using the effective sample-size funnel plot and Egger's test.

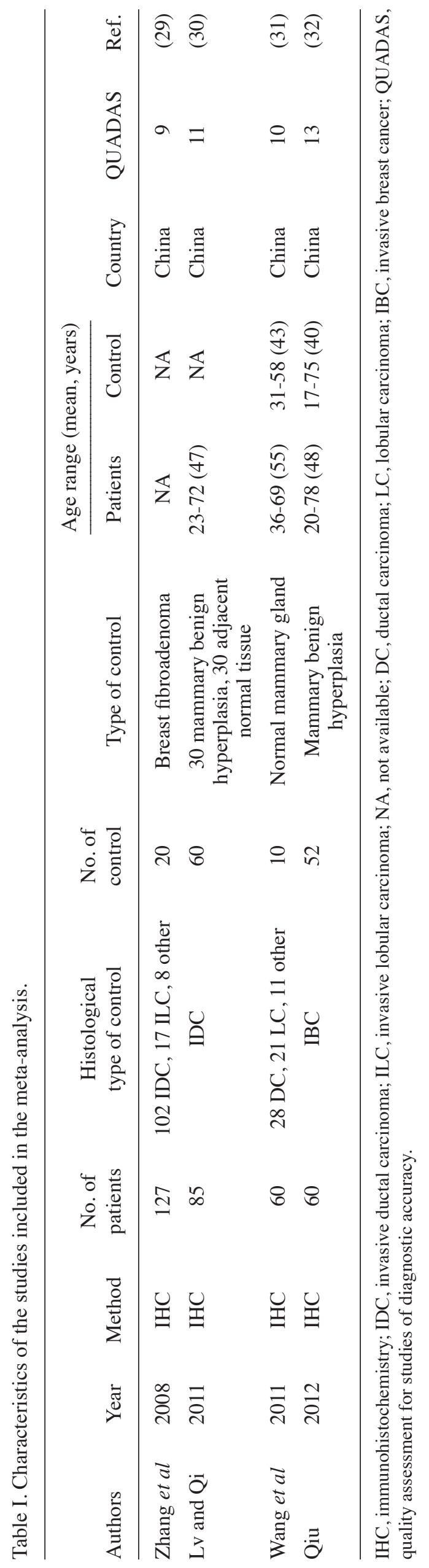




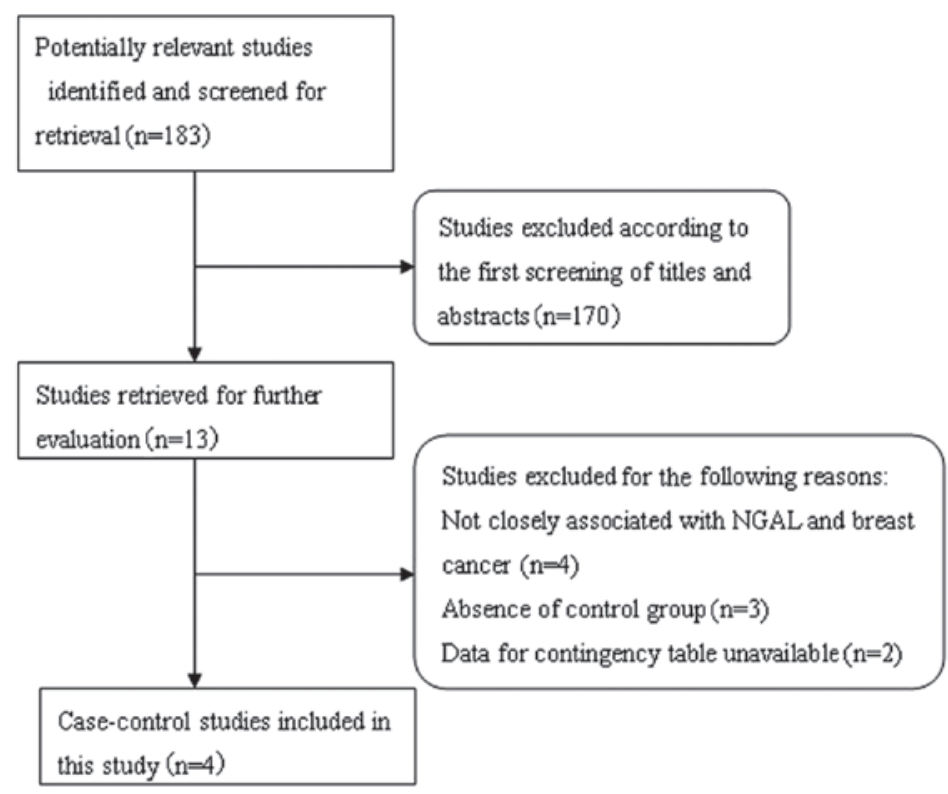

Figure 1. Flow diagram of included/excluded studies.

A

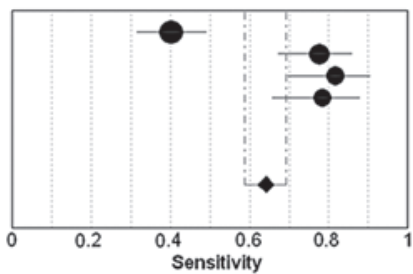

C

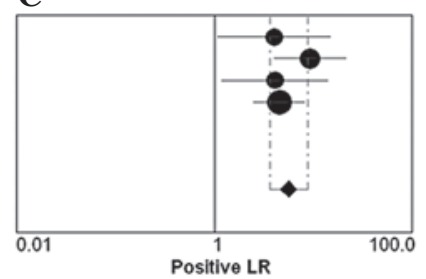

Zhang XF et al.2008 Lv CG et al.2011 Wang XH et al.2011 Qiu JM.2012

Pooled Sensitivity $=0.64$ (0.59 to 0.69 ) Chi-square $=51.95 ; \mathrm{df}=3(\mathrm{p}=0.0000)$ Chi-square $=51.95 ; \mathrm{df}=3(p=0.000$
Inconsistency (1-square) $=94.2 \%$

Sensitivity $(95 \% \mathrm{Cl})$ $\begin{array}{ll}0.40 & (0.32-0.49) \\ 0.78 & (0.67-0.86) \\ 0.82 & (0.70-0.90)\end{array}$ $0.78 \quad(0.66-0.88)$

B

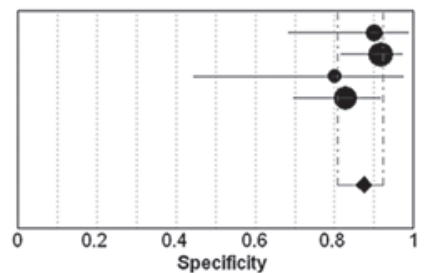

Zhang XF et al.2008 Lv CG et al.2011 Wang XH et al.2011
Qiu JM.2012 Specificity $(95 \% \mathrm{Cl})$ $\begin{array}{ll}0.90 & (0.68-0.99) \\ 0.92 & (0.82-0.97)\end{array}$ $\begin{array}{ll}0.92 & (0.82-0.97) \\ 0.80 & (0.44-0.97)\end{array}$ $0.83(0.70-0.92)$ Pooled Specificity $=0.87(0.81$ to 0.92$)$
Chi-square $=2.62 ; \mathrm{df}=3(\mathrm{p}=0.4533)$ Inconsistency (1-square) $=0.0 \%$

D

Positive LR ( $95 \% \mathrm{Cl})$ Zhang XF et al. 200 Wang XH et al.2011 Qiu JM.2012

\section{Fixed-Effects Model}

Pooled Positive $\mathrm{LR}=5.63(3.63$ to 8.74 ) Cochran- $Q=2.36 ; \mathrm{df}=3(\mathrm{p}=0.5016)$ Inconsistency (1-square) $=0.0 \%$
I $\begin{array}{ll}4.02 & (1.06-15.21 \\ 9.32 & (3.99-21.73\end{array}$ $\begin{array}{ll}9.32 & (3.99-21.73 \\ 4.08 & (1.18-14.19\end{array}$ $\begin{array}{ll}4.08 & (1.18-14.19 \\ 4.53 & (2.46-8.32)\end{array}$

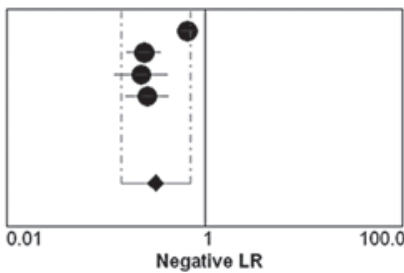

Negative LR $(95 \% \mathrm{Cl}$ Zhang XF et al.2008 V CG et al.2011 Wang XH et al 20
Qiu JM.2012 $0.66 \quad(0.54-0.82)$ $0.24(0.16-0.37)$ $0.23(0.12-0.42)$ Random-Effects Model Pooled Negative LR $=0.32$ ( 0.14 to 0.71 ) Cochran- $Q=48.94 ; \mathrm{df}=3(\mathrm{p}=0.0000)$ Inconsistency (1-square) $=93.9 \%$

Figure 2. Forest plots of the pooled (A) sensitivity, (B) specificity, (C) positive likelihood ratio (LR), (D) negative LR of neutrophil gelatinase-associated lipocalin for the diagnosis of breast cancer. The solid circles represent each individual study and the diamond represents the pooled diagnostic odds ratio. The size of the circle is proportional to the size of the included study. Error bars are $95 \%$ confidence intervals.

Statistical analysis was implemented by MetaDisc 1.4 and Stata 11.0 software.

\section{Results}

Search results and study characteristics. The systematic literature search generated a total of 183 references based on the search strategy. We excluded 170 studies after screening the titles and abstracts, as the majority of these studies did not fulfill the criteria for our meta-analysis, while others were excluded due to duplication or review articles. A careful review of the remaining 13 studies revealed that 4 studies did not focus on the association between NGAL and breast cancer, and were excluded. Subsequently, a further 5 studies were excluded; 3 studies were excluded as they were performed on tissue microarrays and lacked control groups (25-27) and 2 studies were excluded as they did not interpret the IHC results with regard to negative/positive and had insufficient data for constructing the $2 \times 2$ contingency tables $(24,28)$. Following exclusion of the abovementioned studies, 4 studies were included in this meta-analysis (29-32). A flow chart showing the study selection procedure is given in Fig. 1.

A database was established based on the extracted information from these 4 studies (Table I). These 4 studies were single-center trials conducted in China and included 332 breast cancer patients. NGAL expression was analyzed in paraffin sections by IHC. The quality of each study was appraised according to QUADAS. The results are shown in Table I.

Diagnostic accuracy analyses. The forest plot of sensitivity, specificity, PLR, NLR and DOR for NGAL test in breast cancer diagnosing is shown in Figs. 2 and 3. The overall pooled sensitivity and specificity of all studies were 64\% (95\% CI, 0.59-0.69) and $87 \%$ (95\% CI, 0.81-0.92), respectively. The overall pooled 


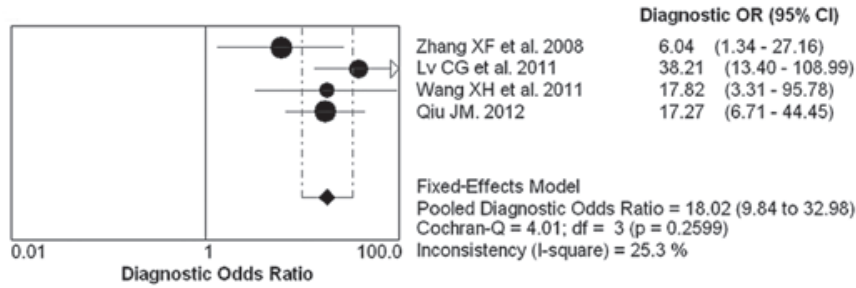

Figure 3. Forest plots of the pooled diagnostic odds ratio of neutrophil gelatinase-associated lipocalin for the diagnosis of breast cancer. The solid circles represent each individual study and the diamond represents the pooled diagnostic odds ratio. The size of the circle is proportional to the size of the included study. Error bars are $95 \%$ confidence intervals.

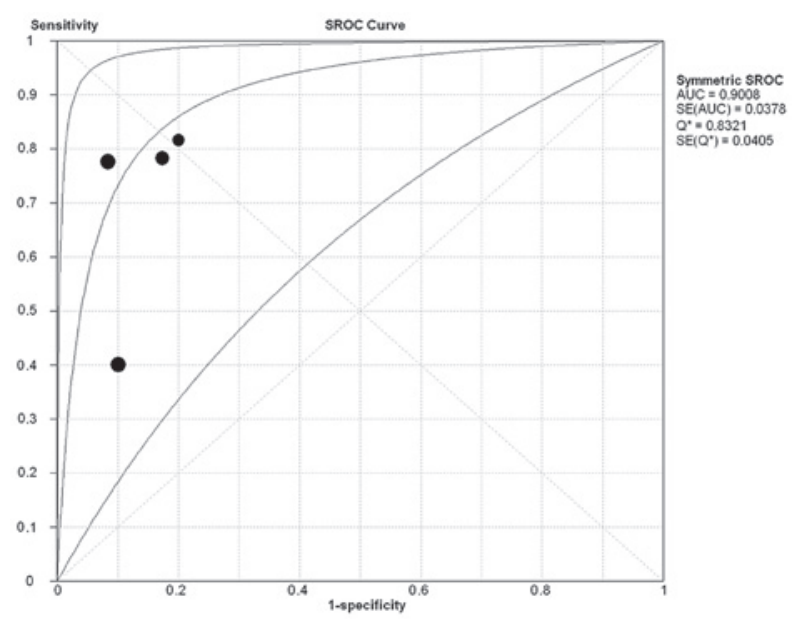

Figure 4. Summary receiver operator characteristic (sROC) curve of all included studies in the diagnosis of breast cancer. The solid circles represent each individual study in the meta-analysis. The size of the circle is proportional to the size of the included study.

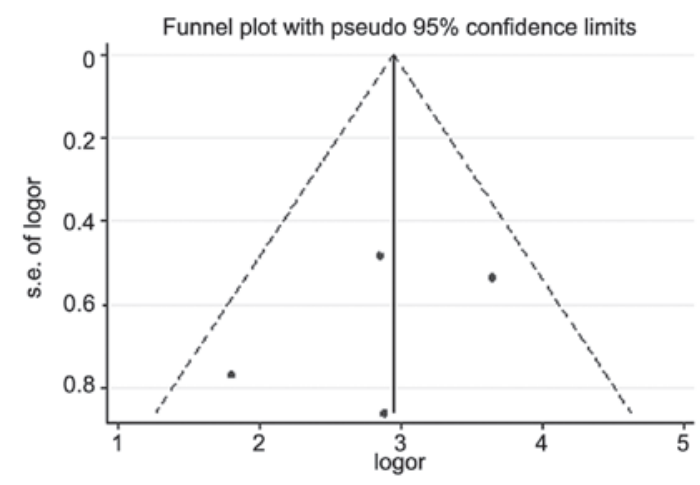

Figure 5. Funnel plot for the assessment of publication bias. The size of circle represents each study in the meta-analysis.

PLR and NLR were 5.63 (95\% CI, 3.63-8.74) and 0.32 (95\% CI, 0.14-0.71). The pooled DOR was 18.02 (95\% CI, 9.84-32.98).

Summary receiver operating characteristics. The sROC curve for NGAL expression showing true-positive rates against false-positive rates from each study demonstrates the trade-off between sensitivity and specificity. The studies were included to construct the sROC curve (Fig. 4). The AUC for the diagnosis of breast cancer was 0.9008 and the $\mathrm{Q}^{*}$-value was 0.8321 .
Test of heterogeneity. A threshold analysis was performed to explore the threshold effect, which was evaluated with the Spearman's correlation coefficient, using the Moses model weighted by inverse variance. Not statistically significant difference was observed (Spearman's correlation coefficient, $0.8, \mathrm{P}=0.2)$.

Cochran's Q test and the $\mathrm{I}^{2}$ statistic were used to evaluate the presence of statistical heterogeneity in the 4 studies examined (Figs. 2 and 3), and the following results were identified: pooled sensitivty (Chi-square $\left.=51.95, \mathrm{I}^{2}=94.2 \%, \mathrm{P}<0.001\right)$, specificity (Chi-square $\left.=2.62, \mathrm{I}^{2}=0, \mathrm{P}>0.05\right), \mathrm{PLR}\left(\right.$ Chi-square $=2.36, \mathrm{I}^{2}=0$, $\mathrm{P}>0.05), \mathrm{NLR}$ (Chi-square $\left.=48.94, \mathrm{I}^{2}=93.9 \%, \mathrm{P}=0.001\right)$ and DOR (Chi-square $\left.=4.01, \mathrm{I}^{2}=25.3, \mathrm{P}>0.05\right)$. By meta-regression analysis, the number of cases in control group or QUADAS score was not the heterogeneity source.

Publication bias. Funnel plot and Egger's test were performed to access the publication bias of these studies. The shape of funnel plots showed symmetry (Fig. 5). The P-value of Egger's test was 0.5 . The result did not suggest any evidence of publication bias.

\section{Discussion}

NGAL is a small, secreted glycoprotein with proposed functions in cell proliferation, survival and morphogenesis. NGAL is expressed in a variety of tumor types including breast cancer (25). In normal human mammary epithelial cells, NGAL expression is under estrogen control (33), while in malignant human mammary epithelial cells, NGAL appears to escape from hormonal regulation, since this protein is most abundant in ER-negative breast cancer cell lines and primary tumor samples (25).

In this study, we clarified the diagnostic accuracy of NGAL for breast cancer by meta-analysis of 4 studies and the results suggested a relationship between NGAL and breast cancer. Using the bivariate model for diagnostic meta-analysis, we found a summary AUC of 0.9008. The DOR is a single indicator used to evaluate the diagnostic value of proposed tests. The pooled DOR of NGAL was 18.02, representative of the odds ratio between breast cancer patients and controls. The pooled sensitivity and specificity were 64 and $87 \%$, respectively, indicating that the assay may result in $36 \%$ false-negative and $13 \%$ false-positive test results. The overall results indicated that NGAL test may be useful in the diagnosis of breast cancer. However, the low sensitivity but high specificity suggested that a patient with a positive result needed to undergo further laboratory evaluation and imaging.

When PLR $>10$ or NLR $<0.1$, the possibility of approving or negating a diagnosis of a disease is significantly increased. In our study, the pooled PLR was 5.63, indicating that the NGAL test was 5.63 times more likely to achieve a correct NGAL-positive test result in the breast cancer group compared with the controls. The pooled NLR was 0.32 , indicating that the possibility of the NGAL test achieving an incorrect NGAL-negative test result in the breast cancer group was 32\% compared with the controls. The overall results indicated that the NGAL protein test had a certain value in the diagnosis of breast cancer. 
The heterogeneity of the 4 studies was analyzed. Results of the Spearman's correlation coefficient indicated that heterogeneity was not correlated with the threshold effect. The factors, number of controls and QUADAS score were added to the meta-regression, but these did not explain the heterogeneity. We hypothesized that the heterogeneity was due to the limited sample size of the 4 selected studies in this meta-analysis.

Limitations of the present meta-analysis should also be considered. First, the number of studies meeting the inclusion criteria was limited as the focus was on China alone. Studies from other countries were excluded due to the absence of control groups (25-27) or a lack of particular data. Second, IHC was utilized for the detection of NGAL in the 4 included primary studies. One study pertaining to NGAL quantified by ELISA assay of blood sample was not included as the information was not interpreted using odds ratios (28). Third, certain studies used a limited sample size. Thus, due to the limitations of this present meta-analysis, more worldwide studies are required to confirm the value of the NGAL test for breast cancer diagnosis in the future.

In summary, the association of NGAL and breast cancer was assessed by pooling the included data via a systematic meta-analysis. The results of the present study demonstrated that NGAL is a potential biomarker for the diagnosis of breast cancer.

\section{References}

1. Gion M, Mione R, Leon AE and Dittadi R: Comparison of the diagnostic accuracy of CA27.29 and CA15.3 in primary breast cancer. Clin Chem 45: 630-637, 1999.

2. Gion M, Mione R, Leon AE, Luftner D, Molina R, Possinger K and Robertson JF: CA27.29: a valuable marker for breast cancer management. A confirmatory multicentric study on 603 cases Eur J Cancer 37: 355-363, 2001.

3. Luftner D, Luke C and Possinger K: Serum HER-2/neu in the management of breast cancer patients. Clin Biochem 36 233-240, 2003.

4. Wang Y, Fang F, Shi C, et al: Evaluation of a method for the simultaneous detection of multiple tumor markers using a multiplex suspension bead array. Clin Biochem 45: 1394-1398, 2012.

5. Nicolini A, Carpi A, Ferrari P and Rossi G: Immunotherapy prolongs the serum CEA-TPA-CA15.3 lead time at the metastatic progression in endocrine-dependent breast cancer patients: a retrospective longitudinal study. Cancer Lett 263: 122-129, 2008

6. Flower DR: The lipocalin protein family: structure and function. Biochem J 318 (Pt 1): 1-14, 1996.

7. Triebel S, Blaser J, Reinke H and Tschesche H: A $25 \mathrm{kDa}$ alpha 2 -microglobulin-related protein is a component of the $125 \mathrm{kDa}$ form of human gelatinase. FEBS Lett 314: 386-388, 1992.

8. Haase M, Bellomo R, Devarajan P, Schlattmann P and Haase-Fielitz A: Accuracy of neutrophil gelatinase-associated lipocalin (NGAL) in diagnosis and prognosis in acute kidney injury: a systematic review and meta-analysis. Am J Kidney Dis 54: 1012-1024, 2009.

9. Kjeldsen L, Johnsen AH, Sengelov H and Borregaard N: Isolation and primary structure of NGAL, a novel protein associated with human neutrophil gelatinase. J Biol Chem 268: 10425-10432, 1993

10. Hu L, Hittelman W, Lu T, et al: NGAL decreases E-cadherinmediated cell-cell adhesion and increases cell motility and invasion through Rac1 in colon carcinoma cells. Lab Invest 89 531-548, 2009.

11. Zhang XF, Zhang Y, Zhang XH, et al: Clinical significance of Neutrophil gelatinase-associated lipocalin (NGAL) expression in primary rectal cancer. BMC Cancer 9: 134, 2009.

12. Kubben FJ, Sier CF, Hawinkels LJ, et al: Clinical evidence for a protective role of lipocalin-2 against MMP-9 autodegradation and the impact for gastric cancer. Eur J Cancer 43: 1869-1876, 2007.
13. Zhang H, Xu L, Xiao D, et al: Upregulation of neutrophil gelatinase-associated lipocalin in oesophageal squamous cell carcinoma: significant correlation with cell differentiation and tumour invasion. J Clin Pathol 60: 555-561, 2007.

14. Friedl A, Stoesz SP, Buckley P and Gould MN: Neutrophil gelatinase-associated lipocalin in normal and neoplastic human tissues. Cell type-specific pattern of expression. Histochem J 31: 433-441, 1999.

15. Li LG, Zhou T and Hou YM: Expression and functional analysis of NGAL gene in human hepatocellular carcinoma. J Fudan Univ (Nat Sci) 51: 91-98, 2012.

16. Iannetti A, Pacifico F, Acquaviva R, et al: The neutrophil gelatinase-associated lipocalin (NGAL), a NF-kappaB-regulated gene, is a survival factor for thyroid neoplastic cells. Proc Natl Acad Sci USA 105: 14058-14063, 2008.

17. Furutani M, Arii S, Mizumoto $\mathbf{M}$, Kato $\mathbf{M}$ and Imamura $\mathbf{M}$ : Identification of a neutrophil gelatinase-associated lipocalin mRNA in human pancreatic cancers using a modified signal sequence trap method. Cancer Lett 122: 209-214, 1998.

18. Laurell H, Bouisson M, Berthelemy P, et al: Identification of biomarkers of human pancreatic adenocarcinomas by expression profiling and validation with gene expression analysis in endoscopic ultrasound-guided fine needle aspiration samples. World J Gastroenterol 12: 3344-3351, 2006

19. Tong Z, Kunnumakkara AB, Wang H, et al: Neutrophil gelatinase-associated lipocalin: a novel suppressor of invasion and angiogenesis in pancreatic cancer. Cancer Res 68: 6100-6108, 2008.

20. Mahadevan NR, Rodvold J, Almanza G, Perez AF, Wheeler MC and Zanetti M: ER stress drives Lipocalin 2 upregulation in prostate cancer cells in an NF- $\kappa \mathrm{B}$-dependent manner. BMC Cancer 11: 229, 2011.

21. Villalva C, Sorel N, Bonnet ML, et al: Neutrophil gelatinase-associated lipocalin expression in chronic myeloid leukemia. Leuk Lymphoma 49: 984-988, 2008.

22. Leng X, Lin H, Ding T, et al: Lipocalin 2 is required for BCR-ABL-induced tumorigenesis. Oncogene 27: 6110-6119, 2008.

23. Jones C, Mackay A, Grigoriadis A, et al: Expression profiling of purified normal human luminal and myoepithelial breast cells: identification of novel prognostic markers for breast cancer. Cancer Res 64: 3037-3045, 2004.

24. Yang J, Bielenberg DR, Rodig SJ, et al: Lipocalin 2 promotes breast cancer progression. Proc Natl Acad Sci USA 106: 3913-3918, 2009.

25. Bauer M, Eickhoff JC, Gould MN, Mundhenke C, Maass N and Friedl A: Neutrophil gelatinase-associated lipocalin (NGAL) is a predictor of poor prognosis in human primary breast cancer. Breast Cancer Res Treat 108: 389-397, 2008.

26. Wenners AS, Mehta K, Loibl S, et al: Neutrophil gelatinase-associated lipocalin (NGAL) predicts response to neoadjuvant chemotherapy and clinical outcome in primary human breast cancer. PLoS One 7: e45826, 2012

27. Stoesz SP, Friedl A, Haag JD, Lindstrom MJ, Clark GM and Gould MN: Heterogeneous expression of the lipocalin NGAL in primary breast cancers. Int J Cancer 79: 565-572, 1998.

28. Provatopoulou X, Gounaris A, Kalogera E, et al: Circulating levels of matrix metalloproteinase-9 (MMP-9), neutrophil gelatinase-associated lipocalin (NGAL) and their complex MMP-9/NGAL in breast cancer disease. BMC Cancer 9: 390, 2009.

29. Zhang XF, Zhou SM, Wan L, et al: Relationship between NGAL expression and progression, metastasis and prognosis of breast cancer. J Pract Oncol 23: 206-210, 2008.

30. Lv CG and Qi FJ: Expression of NGAL and MMP-9 protein in invasive breast cancer and its clinical application. Guangdong Med J 32: 1287-1289, 2011

31. Wang XH, Guo YY and Gou XM: Expression of NGAL in breast cancer and its clinical application. China Pract Med 6: 43-45, 2011.

32. Qiu JM: Clinical significance of determination in tumor cells NGAL levels of breast cancer patients with immuno-histochemistry. J Radioimmunol 25: 546-548, 2012.

33. Stuckey R, Aldridge T, Lim FL, et al: Induction of iron homeostasis genes during estrogen-induced uterine growth and differentiation. Mol Cell Endocrinol 253: 22-29, 2006. 